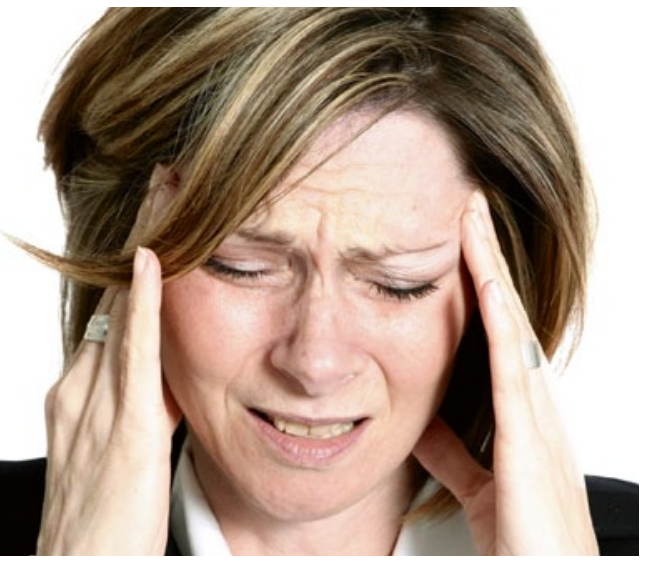

\title{
Keine Hirnatrophie durch chronische Schmerzen
}

\author{
Bei chronischen Schmerzen verändert sich auch das Gehirn: \\ Manche Hirnarale werden dünner, sodass bleibende \\ Schäden befürchtet werden. Allerdings sind diese Verän- \\ derungen reversibel: Nach einer erfolgreichen Schmerz- \\ therapie normalisiert sich das Volumen.
}

\begin{abstract}
Eine Verminderung der grauen Substanz lässt sich bei fast allen Patienten mit chronischen Schmerzen beobachten - egal ob Kopf-, Rücken- oder Phantomschmerzen, erläuterte Prof. Arne May, Uniklinikum Hamburg-Eppendorf. Bekannt sind aus der strukturellen Bildgebung ausgedünnte Areale im Bereich des vorderen Cingulums, der Insula, im Hirnstamm oder S2-Bereich. Lange Zeit befürchtete man, dass es sich um eine irreversible Atrophie mit Beeinträchtigung der kognitiven Funktionen handelt. Hier gibt es nun Entwarnung: Die hirnstrukturellen Veränderungen sind bei einer erfolgreichen Schmerztherapie offenbar vollkommen reversibel. May nannte als Beispiel eine Studie mit 14 Rückenschmerzpatienten, die sich einer interventionellen Therapie unterzogen. Die Patienten wurden vor dem Eingriff und sechs Monate danach per strukturelle MRT untersucht. Dabei zeigten sich anfangs die bekannten kortikalen Ausdünnungsmuster. Zusätzlich absolvierten die Patienten Kognitions-
\end{abstract}

tests während der fMRT. Bei Aufmerksamkeitsübungen fiel eine abnorme Aktivität im ebenfalls etwas geschrumpften linken dorsolateralen präfrontalen Kortex auf. Das überraschende Ergebnis: Ein halbes Jahr später hatten sich die zuvor ausgedünnten Areale wieder verdickt - aber nur bei den Patienten, die tatsächlich deutlich weniger Schmerzen hatten. Diese zeigten nun auch eine normale präfrontale Aktivität in der funktionellen Bildgebung. Dies deute darauf, so May, dass chronischer Schmerz nicht zu einer neuronalen Atrophie führt. Am ehesten ließen sich die Veränderungen durch neuronale Plastizität erklären. Demnach würde eine Mindernutzung bestimmter Areale die beobachteten Schrumpfungen auslösen, eine vermehrte Nutzung diese dann wieder rückgängig machen. Thomas Müller, Springer Medizin

Neuro Update, Mainz, 15.2.2013. Vortrag „Schmerz/Kopfschmerz" von Prof. Arne May, Uniklinikum Hamburg-Eppendorf

\section{Gefäßstenosen im Gehirn}

\section{Stents bleiben eine Option}

\author{
Intrakranielle Stents können bei Patienten mit hohem Schlaganfallrisiko von Vorteil sein. Etwa \\ dann, wenn die Stenose symptomatisch ist und sich zugleich gut erreichen lässt.
}

Die 2011 veröffentlichte Studie SAMMPRIS enttäuschte die Befürworter der Stenttherapie: Patienten mit einer intrakraniellen Stenose (Gefäßverschluss über $70 \%$ ) erhielten ein aggressives medikamentöses Management (Clopidogrel plus ASS) mit oder ohne zusätzlichen Stent. Mit Stent kam es in den ersten 30 Tagen fast dreimal so häufig zu Todesfällen und Schlaganfällen wie mit medikamentöser Therapie allein (14,7\% vs. 5,8\%).

Das ist aber nicht das Ende der Stenttherapie bei Gefäßverschlüssen im Gehirn, so Prof. Peter Ringleb, Uniklinikum Heidelberg. Er wies darauf hin, dass in SAMMPRIS nicht geschaut wurde, ob die Stenosen hämodynamisch relevant waren. StenosePatienten mit Kollateralen haben nur ein sehr geringes Schlaganfallrisiko - für sie sei das medikamentöse Management sicher die bessere Wahl. Zudem gab es die meisten Schlaganfälle und Hirnblutungen am Tag des Eingriff. Zum Teil lasse sich dies damit er- klären, dass beim Einführen des Stents Material aus der Stenose verschoben wird und es zum Verschluss der Perforatoren kommt. Solche Probleme ließen sich möglicherweise mit selbstexpandierenden Stents reduzieren. Für Ringleb ist die Stentimplantation also weiterhin eine Option, und zwar dann, wenn die Stenose hämodynamisch relevant ist und trotz intensiver medikamentöser Therapie immer wieder Symptome verursacht. Als weitere Voraussetzung sollte die Stenose technisch leicht zugänglich sein, um perioperative Komplikationen zu vermeiden. Zudem, so Ringleb, müssen die Patienten für den Eingriff ausreichend stabilisiert sein, nach Schlaganfall oder TIA sei ein Mindestabstand von einer Woche zu empfehlen.

Thomas Müller, Springer Medizin

Präsidentensymposium: Schwierige Entscheidungen in der Intensivmedizin bei Schlaganfallpatienten, ANIM, 24.1.2013 in Mannheim 